\title{
VARIABILITY MEASURES IN NEURAL SYSTEMS: BASES FOR AN AUTOMATIC SLEEP STAGING INDEPENDENT OF SPECTRAL ANALYSIS
}

\author{
André Fonseca ${ }^{1}$ and Birajara Soares Machado ${ }^{2}$ \\ ${ }^{1}$ Centro de Matemática, Computação e Cognição, Universidade Federal do ABC, \\ Av. dos Estados, 5001, Santo André, Brazil, 09210580* \\ ${ }^{2}$ Instituto do Cérebro, Hospital Israelita Albert Einstein, \\ Av. Albert Einstein, 627, São Paulo, Brazil, 05652900 ${ }^{\dagger}$
}

\begin{abstract}
In this work we introduce some nonlinear neural dynamics techniques and analyze the performance of two variability measures related to the system uncertainty and predictability in the context of rats sleep study. We propose the bases of a novel automatic sleep staging procedure that is independent of the spectral analysis and can be extended to human sleep EEG data with no need of simultaneous recordings as fMRI, EOG, EMG or polysomnograms.
\end{abstract}

Keywords: neural dynamics, time series analysis, dynamical systems applications, sleep staging.

\section{INTRODUCTION}

From 1985 to 1990, considering the existence of an underlying attractor set that generates the neural activity, several researches started to apply dynamical systems analysis to neural data through the phase space reconstruction process defined by Takens and Whitney [1]. Classical invariants as Lyapunov exponents, fractals dimensions and entropies were estimated from Electroencephalographs (EEG) and local field potentials (LFP), propagating the idea that "brain and chaos" were a perfect match. After 1990 the concept of a "chaotic brain" were reexamined with validation tests. Some authors criticized and rejected previous results and, nowadays, the nonlinear dynamical systems analysis is used not to characterize chaos in neural signals but rather to develop novel variability measures capable of identifying phase space transitions expected in well-defined experiments which results can be validated with hypothesis tests and the noise effect can be discarded [2].

The electrophysiological sleep study in mammals started in 1937 with the characterization of different sleep stages associated to specific brain activity [3].

Sleep can be divided in two categories: nonrapid eye movement (NREM) and rapid eye movement (REM) sleeps. Based on neural responses, NREM has 3 stages. In this work we denote the first two as simply NREM and stage 3 as NREM - slow wave sleep (SWS). SWS is defined by oscillations with high amplitude and frequency less than $4 \mathrm{~Hz}$ (delta wave) [4].

NREM and REM occur in alternating cycles and, among several analysis of their importance, there is the evidence that sleep promotes a fundamental role in memories long-term consolidation associated to neocortex and hippocampus areas, where declarative memory is related to NREM-SWS and procedural memory is related

\footnotetext{
* corresponding author:andre.fonseca@ufabc.edu.br

†birajara@einstein.br
}

to REM sleep [5].

Considering this context, in this work we analyzed the variability of a LFP signal from the CA1 region of Ammon's horn (central structure of the hippocampus) of rats, during NREM, NREM-SWS and REM sleeps and introduce two promising techniques for automatic sleep staging independent of spectral analysis and with high accuracy (compared with visual inspection results).

In section II we present some information of the empirical data used. In section III we introduce the phase space reconstruction and define two variability measures related to the signal uncertainty and regularity. We show their results to a noise and transient simulated signal and, in section IV we point out the variability analysis to the empirical signals. In section $\mathrm{V}$ we discuss the findings and its applicability.

\section{MATERIALS}

Rats, electrodes and surgery description as well as behavioral and eletrofisiologic reports for sleep-wake cycle visual inspection can be found in $[6](\mathrm{PhD}$ thesis in portuguese). The signals sample rate is $500 \mathrm{~Hz}$ and they were recorded during 2 hours (3, 600, 000 time points).

\section{METHODS}

\section{A. Phase Space Reconstruction}

The first step to apply dynamical systems tools in neural data is to consider the signal as a projection of a multi-dimensional underlying attractor and retrieve its geometrical and analytical proprieties by a reconstruction procedure, based on Takens-Whitney delay embedding theorem [1]. Given the scalar signal: $X=$ $\left\{x_{1}, \ldots, x_{n}\right\}$, we reconstruct its corresponding phase space $\left\{\overrightarrow{x_{i}}=\left(x_{i}, x_{i+\tau}, \ldots, x_{i+(m-1) \tau}\right) ; i=1, \ldots, n-(m-1) \tau\right\}$, where $m$ is the embedding dimension and $\tau$ is the time delay related, respectively, to the attractor geometry 
and time scale. Usually, the optimal values $m$ and $\tau$ are estimated from independent procedures although their correlation is not negligible. We have employed the Gautama-Mandic-Hulle method [7, 8] that determines those two parameters jointly. For instance, in figure 1 we present: $(a)$ a solution for the Lorenz system $(\dot{x}=\sigma(y-x), \dot{y}=x(\rho-z)-y, \dot{z}=x y-\beta z ;$ with $\sigma=10, \rho=28$ and $\beta=8 / 3)$ as the underlying dynamics ; $(b)$ its additive noise projection and $(c)$ the reconstructed attractor. Notice that the reconstructed phase space preserves the shape (geometrical and topological features) of the original system.

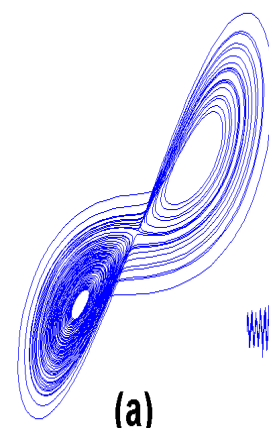

(a)

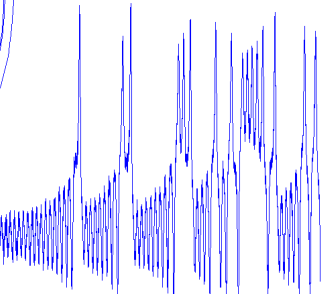

(b)

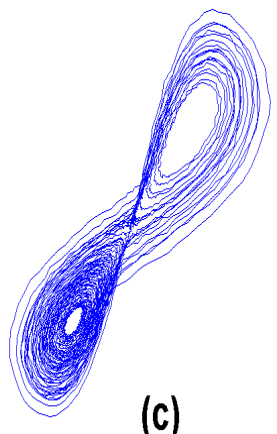

(c)
FIG. 1: Lorenz system reconstruction. (a) 3d signal integrated with step 0.01 and 5,000 points; (b) 1d projection with additive noise; (c) reconstructed attractor with parameters $m=3$ and $\tau=5$.

\section{B. Sample Entropy}

The first variability measure used is the Sample Entropy (SE) [9], which is related to the system uncertainty. For each vector $\overrightarrow{x_{i}}$ defined before, given an embedding dimension $m$, we define its $\varepsilon$-recurrence set $R_{i}=\left\{\overrightarrow{x_{i}}:\left\|\overrightarrow{x_{i}}-\overrightarrow{x_{j}}\right\|<\varepsilon\right\}$, where $\varepsilon$ is the recurrence tolerance. The probability distribution in the reconstructed phase space is $P_{i}=\#\left(R_{i}\right) /(n+(m-1) \tau)$, where \# is the set cardinality. Its mean recurrence probability is $\overline{P_{m}}=\left\langle P_{i}\right\rangle$ and, considering this quantity for embedding dimension $m+1$, we define the Sample Entropy as $S E(m, \tau, \varepsilon)=-\ln \left(\overline{P_{m+1}} / \overline{P_{m}}\right)$, which is related to the loss of information for successive dimensions of the reconstructed phase space.

\section{Cross Prediction Error}

The second variability measure used is the Cross Prediction Error (CPE) [10], which is related to the system predictability. We split the scalar signal $X$ into $n / l$ successive disjoint $l$-length windows $W_{i}=$ $\left\{x_{(i-1) l+1}, \ldots, x_{i l}\right\}$. For each pair $W_{i}$ (input space) and $W_{j}$ (output space) ; $i, j=1, \ldots, n / l$ we reconstruct the phase spaces $\overrightarrow{x_{k}}=\left(x_{k-m+1}, \ldots, x_{k}\right)$ from $W_{i}$ and $\overrightarrow{y_{k}}=\left(y_{k-m+1}, \ldots, y_{k}\right)$ from $W_{j}$, with embedding dimension $m$ and time lag $\tau=1$. To estimate $y_{k+1}$ in the output space using $W_{i}$, we define the estimator $\hat{y}_{k+1}^{x}=\frac{1}{\# S_{\varepsilon}^{x}\left(\overrightarrow{y_{k}}\right)} \sum_{\overrightarrow{x_{p}} \in S_{\varepsilon}^{x}} x_{p+1}$, where $S_{\varepsilon}^{x}\left(\overrightarrow{y_{k}}\right)$ is the ensemble of vectors formed from the input space that lie in the $\varepsilon$-neighbourhood of $\overrightarrow{y_{k}}$, that is, $S_{\varepsilon}^{x}=\left\{\overrightarrow{x_{p}}:\left\|\overrightarrow{x_{p}}-\overrightarrow{y_{k}}\right\|<\varepsilon\right\}$. In the case where $\overrightarrow{y_{k}}$ has no neighbors in the input space, that is, $\# S_{\varepsilon}^{x}\left(\overrightarrow{y_{k}}\right)=0$, we set $\hat{y}_{k+1}^{x}=\langle x\rangle$.

The CPE matrix is defined as:

$$
\gamma_{i, j}=\gamma\left(W_{i}, W_{j}\right)=\sqrt{\frac{1}{l-m} \sum_{k=m}^{l-1}\left(\hat{y}_{k+1}^{x}-y_{k+1}\right)^{2}} .
$$

$\gamma(i, j)$ is a measure of how much the locally reconstructed dynamics from one subset is accurate to predicts another subset. Generally, $\gamma(i, j) \neq \gamma(j, i)$. Therefore pattern changes in the two dimensional CPE matrix indicate changes in the dynamics as phase space transitions [11].

In figure 2 one can observe a simulated noise signal with 3 phase space transitions not detected by time frequency analysis (fast Fourier transform) due to short time series limitations. In other hand, Sample Entropy (red curve) and the CPE matrix were sensible to those transitions.

In this work we extracted from the CPE matrix, for each interval $i=2, \ldots,(n / l)-1$, the mean $\mathrm{CPE}$ value $\frac{1}{3}\left[\gamma_{i, i-1}+\gamma_{i, i}+\gamma_{i, i+1}\right]$, which takes in account the transition from neighbor windows and the auto prediction error. We define this measure as Local Predictability Error (LPE).

\section{RESULTS}

We used 2 hours $500 \mathrm{~Hz}$ LFP signals from the CA1 region of Ammon's horn (central structure of the hippocampus) of 3 rats [6].

Using the Gautama-Mandic-Hulle method [7] we obtained $m=2$ and $\tau=1$ and we set $\varepsilon=0.25$ based on the results found in [11].

We split the signals into 250 windows of size $l=14400$ time points. Then we evaluated the two variability measures, Sample Entropy and Local Predictability Error and normalized its values to the interval $[0,1]$.

From the visual sleep staging for one random chosen rat, we determine correspondents intervals in SE and LPE correlated to NREM, NREM-SWS and REM sleeps. Using those relations we defined an automatic criteria. Then we stage all rats signals using this criteria and compare with their respective visual sleep inspections. In figure 3 we show the results for one rat.

The automatic sleep staging accuracy and Cohen's kappa coefficient for the Sample Entropy and Local Predictability Error are in table I. 


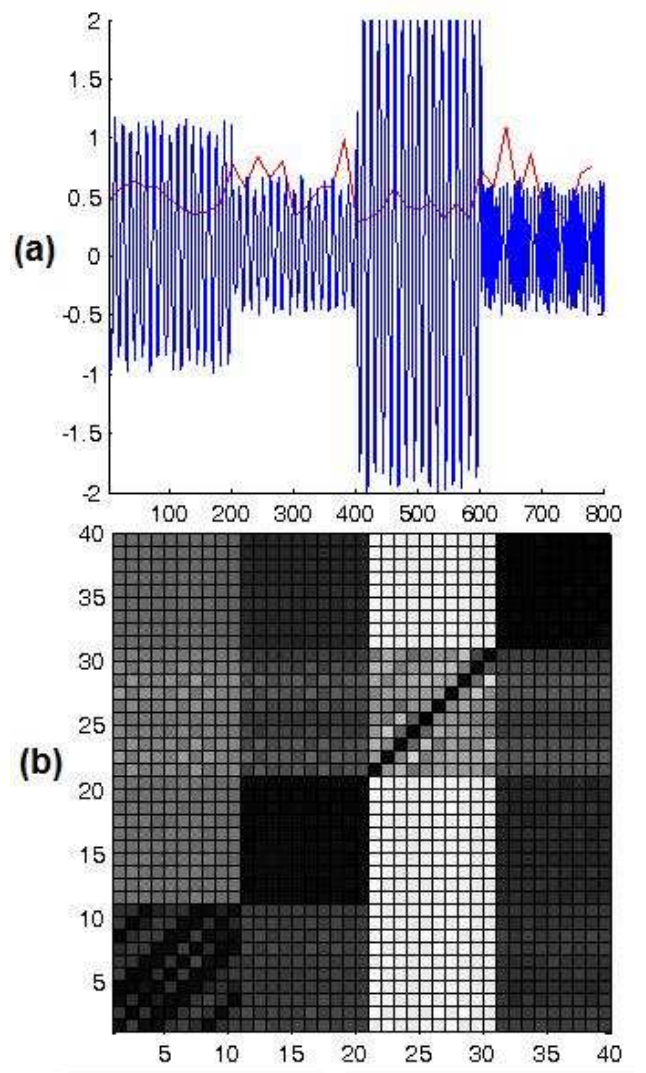

FIG. 2: Simulated noise signal analysis. (a) signal (blue) and Sample Entropy (red) for $m=2, \tau=1$ and $\varepsilon=0.25$. (b) CPE matrix in gray scale (from black low value to white high value) with same parameters as (a) and $l=20$ time points.

\begin{tabular}{|c|c|c|}
\hline & Accuracy & Kappa \\
\hline SE & $0.82 \pm 2.10$ & $0.67 \pm 0.12$ \\
\hline LPE & $0.83 \pm 3.10$ & $0.65 \pm 0.25$ \\
\hline
\end{tabular}

TABLE I: Automatic sleep staging accuracy and kappa index

\section{CONCLUSION}

In figure 3 we can notice a high correlation between sleep cycles exchanges and SE and LPE. The phase space transitions expected in sleep study are detected by SE (uncertainty measure) with higher values for NREM sleep and lower values for REM sleep. The same transitions are also detected by LPE (predictability measure) but in a opposite manner. The NREM-SWS sleep has intermediary values in both measures.

All measures were computed for others $m, \tau$ and $\varepsilon$ parameters, close to the ones cited in section IV with similar results. This fact establish a robustness against parameters for both techniques.

Those measures are independent of spectral analysis, have high accuracy and, specifically in sleep study, the difficult in dealing with theta and gamma bands cycles was avoided $[4,6]$. This novel approach is sensible to

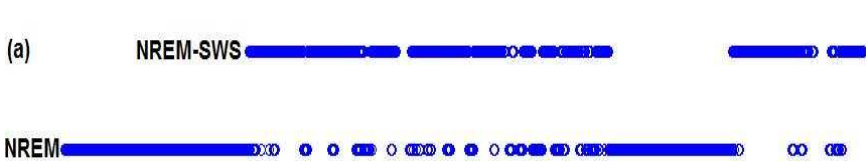

(b)
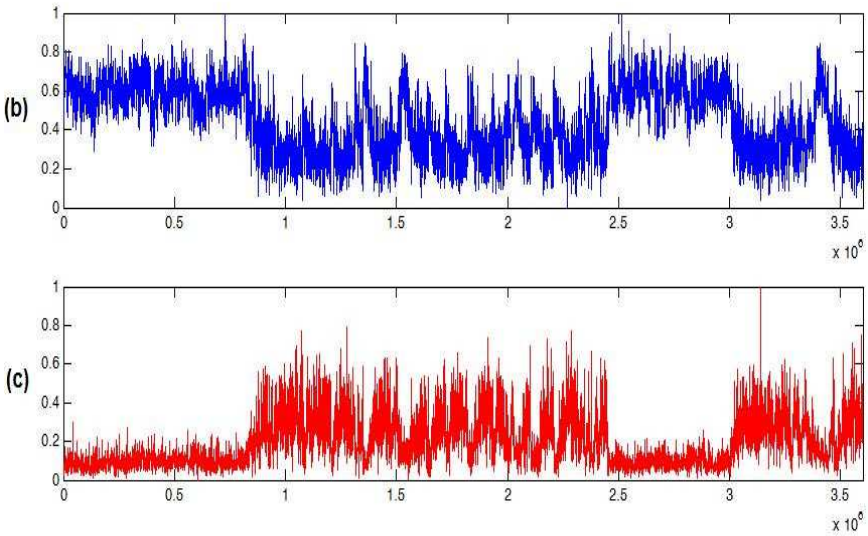

FIG. 3: Variability measures for a CA1 LFP signal. (a) Visual sleep staging; (b) Sample Entropy; (c) Local Predictability Error.

non-linear and non-stationary data $[9,10]$ and it is possible to combine those two procedures, Sample Entropy and Local Predictability Error, to build a new automatic sleep staging procedure.

\section{Acknowledgement}

We would like to thank Dr. E. E. Schenberg [6] for the data and previous analysis.
[1] Floris Takens, "Detecting strange attractors in turbulence", Dynamical Systems and Turbulence, Lecture Notes in Mathematics, 898 (1981).

[2] C.J. Stam, "Nonlinear dynamical analysis of EEG and MEG: Review of an emerging field", Clinical Neurophysiology, 116 (2005).
[3] H. Blake, and R.W. Gerard,"Brain potentials during sleep", American Journal of Physiology, 119 (1937).

[4] Robert L. Sack et al, "Circadian Rhythm Sleep Disorders: Part II, Advanced Sleep Phase Disorder, Delayed Sleep Phase Disorder, Free-Running Disorder, and Irregular Sleep-Wake Rhythm", Sleep, 30 (2007). 
[5] Robert Stickgold, "Sleep-dependent memory consolidation", Nature, 437 (2005).

[6] E. E. Schenberg, "Caracterização eletrofisiológica da circuitaria hipocampal durante o ciclo sono-vigília do rato", PhD Thesis, Universidade de São Paulo (2010).

[7] Temujin Gautama, Danilo P. Mandic, and Marc M. Van Hulle, A differential entropy based method for determining the optimal embedding parameters of a signal, Acoustics, Speech, and Signal Processing, 2003.

[8] L. F. Kozachenko, N. N. Leonenko. "Sample Estimate of the Entropy of a Random Vector". Problemi Peredachi Informatsii, 1987
[9] Joshua S. Richman, and J. Randall Moorman, "Physiological time-series analysis using approximate entropy and sample entropy", American Journal of Physiology Heart and Circulatory Physiology, 278 (2000).

[10] Thomas Schreiber, "Detecting and analyzing nonstationarity in a time series using nonlinear cross predictions", Physical Review Letters, 78 (1997).

[11] G. Baggio, and A. Fonseca, "Complex dynamics for semantic memory access in reading", Journal of the Royal Society Interface, 9 (2011). 